\title{
Towards a Safe Lithium-Sulfur Battery with a Flame-Inhibiting Electrolyte and a Sulfur-Based Composite Cathode**
}

\author{
Jiulin Wang,* Fengjiao Lin, Hao Jia, Jun Yang, Charles W. Monroe, and Yanna NuLi
}

\begin{abstract}
Of the various beyond-lithium-ion batteries, lithium-sulfur (Li-S) batteries were recently reported as possibly being the closest to market. However, its theoretically high energy density makes it potentially hazardous under conditions of abuse. Therefore, addressing the safety issues of Li-S cells is necessary before they can be used in practical applications. Here, we report a concept to build a safe and highly efficient Li-S battery with a flame-inhibiting electrolyte and a sulfurbased composite cathode. The flame retardant not only makes the carbonates nonflammable but also dramatically enhances the electrochemical performance of the sulfur-based composite cathode, without an apparent capacity decline over 750 cycles, and with a capacity greater than $800 \mathrm{~mA} \mathrm{~h}^{-1} \mathrm{~g}^{-1}$ (sulfur) at a rate of $10 \mathrm{C}$.
\end{abstract}

Li-S batteries, with a remarkably high theoretical energy density of $2567 \mathrm{Wh} \mathrm{kg}^{-1}$ compared to those with other electrode materials and a high specific capacity of $1672 \mathrm{~mA} \mathrm{~h}^{-1} \mathrm{~g}^{-1}$ compared to batteries with elemental sulfur cathodes, have recently attracted intense interest. ${ }^{[1]}$ As a consequence of the abundance of sulfur resources and the low cost and nontoxicity of Li-S batteries, they show great promise for large-scale applications in renewable energy fields. Currently, of the various beyond-lithium-ion systems, Li-S batteries are believed to be the closest to market. ${ }^{[2]}$ Compared to the insertion chemistry of conventional lithium-ion batteries, Li-S batteries utilize an "integration chemistry" that reduces the possibility of self-ignition. ${ }^{[3]}$ Nonetheless, as a key aspect of any energy storage device, including batteries, the safety issues of Li-S cannot be ignored $^{[4]}$ because 1) the lithium dendrite formed during cycling is highly active; 2) the mixtures of conductive carbon and sulfur are flammable and explosive; and 3) the high volatility and flammability of carbonates or ethers make them thermally unstable ${ }^{[5]}$ and prone to ignition. Li-S batteries,

[*] Prof. J. L. Wang, F. J. Lin, H. Jia, Prof. J. Yang, Prof. Y. N. NuLi School of Chemistry and Chemical Engineering

Shanghai Jiao Tong University

Shanghai 200240 (China)

E-mail: wangjiulin@sjtu.edu.cn

Prof. C. W. Monroe

Department of Chemical Engineering, University of Michigan Ann Arbor, MI 48109 (USA)

[**] This work was supported by the National Natural Science Foundation of China $(51272156,21333007$, and 21273146) and SJTU-UM joint research project. We also thank the Instrumental Analysis Center of Shanghai Jiao Tong University for XPS measurements.

$\square$ Supporting information for this article is available on the WWW under http://dx.doi.org/10.1002/anie.201405157. which have a high theoretical energy density comparable to that of trinitrotoluene (TNT) ${ }^{[6]}$ might cause serious hazards in terms of fire or explosion when exposed to flames. Therefore, addressing the safety issues of Li-S batteries is necessary to accelerate their practical applications as largescale energy storage and conversion devices or as miniature power sources for wearable products.

The flammability of solvents can be eliminated through the use of either flame-retardant additives in standard electrolytes ${ }^{[7]}$ or an inherently nonflammable electrolyte, such as a solid-state electrolyte, silanes, or an ionic liquid. ${ }^{[8]}$ The former strategy of adding flame retardants to organic electrolytes as cosolvents or additives is an economical option. Four main types of flame retardants have been explored in lithium-ion battery electrolytes: phosphates, phosphides, phosphazene, and fluorinated ethers. ${ }^{[9]}$ In general, the amount of additive required to achieve nonflammability significantly deteriorates the electrochemical performance of lithium-ion batteries. Furthermore, some additives are not compatible with the electrode materials. However, little information about the inner or outer protection of Li-S batteries has been reported to date. On the basis of the above considerations, we propose a concept to build safe Li-S batteries with both a flame-inhibiting electrolyte and a nonflammable cathode.

The conventional electrolyte which contains ethylene carbonate (EC) and dimethyl carbonate (DMC) solvents is highly flammable. Figure $1 \mathrm{a}$ indicates that the combustion of this electrolyte lasts for $14 \mathrm{~s}$. The addition of tris(2,2,2trifluoroethyl) phosphite (TTFP), with a boiling point of approximately $131^{\circ} \mathrm{C}$, results in the EC- and DMC-based electrolyte becoming nonflammable (Figure 1b). Figure $1 \mathrm{e}$ demonstrates the effect of the TTFP content on the selfextinguishing time (SET) and ionic conductivity of the electrolyte. The SET of the electrolyte decreased sharply from $104.08 \mathrm{~s} \mathrm{~g}^{-1}$ for the electrolyte to $61.50 \mathrm{~s} \mathrm{~g}^{-1}$ after $5 \mathrm{wt} \%$ TTFP was added, then further to $11.4 \mathrm{sg}^{-1}$ after $10 \mathrm{wt} \%$ TTFP was added. The SET value decreased to zero when the content of TTFP was increased to $15 \mathrm{wt} \%$. Concentrations of TTFP greater than $10 \mathrm{wt} \%$ can effectively retard or even eliminate flammability in electrolyte solutions. However, the conductivity of the electrolytes exhibited a linear decrease with increasing TTFP content, which might be due to the relatively high viscosity $\left(17.7 \mathrm{~mm}^{2} \mathrm{~s}^{-1}\right.$ at $\left.20^{\circ} \mathrm{C}\right)$ and low dielectric constant of TTFP. ${ }^{[7 b]}$

Two mechanisms have been suggested to explain the effect of flame-retardant additives. ${ }^{[10]}$ One is a physical charforming process, which builds up an isolating layer between the condensed and gas phases; the other is chemical radical termination of the combustion process. For organic liquid 

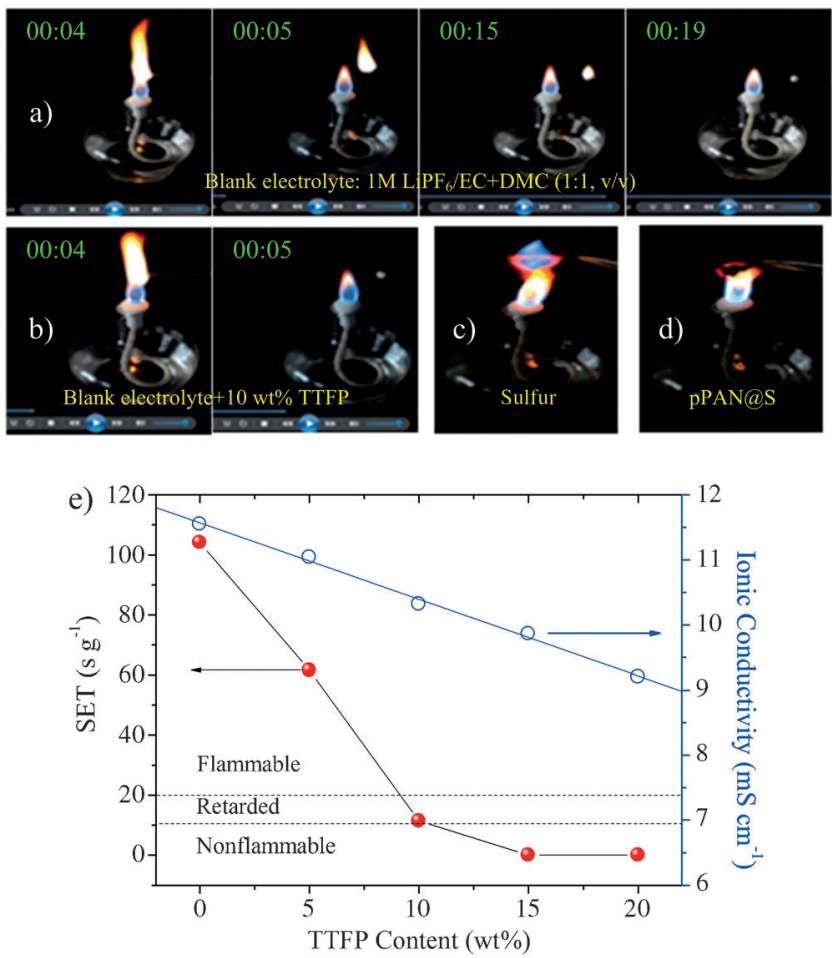

Figure 1. Flame tests and the effect of TTFP on the SET. a) Blank electrolyte; b) flame-inhibiting electrolyte; c) elemental sulfur in the flame; d) PPAN@S in the flame; e) SET and conductivity of the electrolytes with different TTFP contents.

electrolytes, the main flammability mechanism is the latter. When TTFP is heated to temperatures above the pyrolysis temperature, it generates free radicals ( $\mathrm{PO}^{*}$ and $\mathrm{F}^{*}$ ), which are able to actively capture other free radicals emitted by the burning electrolyte (usually $\mathrm{H}^{\cdot}$ and $\left.{ }^{\circ} \mathrm{OH}\right)$ to retard the combustion. ${ }^{[7]}$

Elemental sulfur, which is the main component in explosive materials, is well known to be highly flammable and to exhibit a light-blue flame during combustion (Figure 1c). Furthermore, polyacrylonitrile (PAN) is generally classified as a flammable textile material by virtue of its low limited oxygen index (LOI) value of approximately $18 \% .{ }^{[11]}$ However, their reaction products, pyrolyzed polyacrylonitrile and sulfur (pPAN@S) composite with a sulfur content of $43 \mathrm{wt} \%$, are nonflammable and neither electrolyte; d) rate performance. melt nor shrink when left in a flame (Figure 1d). PAN was dehydrogenated by sulfur at approximately $300^{\circ} \mathrm{C}$ in an inert atmosphere, thereby forming a conductive main chain with an electronic conductivity of about $10^{-4} \mathrm{~S} \mathrm{~cm}^{-1}$, which represents an approximate $10^{26}$-fold increase from that of its insulating precursor. ${ }^{[12]}$ The highly polar $\mathrm{CN}$ functional group cyclized to form a thermally stable heterocyclic compound in a process similar to PAN preoxidation in air. ${ }^{[5,13]}$ This conductive pPAN, with a LOI greater than $31 \%$, is nonflammable, insoluble ${ }^{[14]}$ and serves as an excellent flame retardant for sulfur in the pPAN@S composite.

The theoretical specific capacity of the pPAN@S composite material is $719 \mathrm{~mA} \mathrm{~h}^{-1} \mathrm{~g}^{-1}$, as calculated by multiplying $1672 \mathrm{~mA} \mathrm{~h}^{-1} \mathrm{~g}^{-1}$ by the sulfur content of $43 \mathrm{wt} \%$. The charge and discharge behaviors of the cells in the electrolytes containing different amounts of TTFP are demonstrated in Figure $2 \mathrm{a}$. The addition of TTFP hardly affected the charge/ discharge curves of the pPAN@S composite cathode material, which delivered an initial discharge capacity of about $774 \mathrm{~mA} \mathrm{~h}^{-1} \mathrm{~g}^{-1}$ (composite) (calculated based on the whole composite, including pPAN) or about $1800 \mathrm{~mA} \mathrm{~h}^{-1} \mathrm{~g}^{-1}$ (sulfur) (calculated based on sulfur), thus exceeding the theoretical value of $1672 \mathrm{~mA} \mathrm{~h}^{-1} \mathrm{~g}^{-1}$ for sulfur. This extra capacity may partially derive from lithium insertion into the anionic conjugated backbone in pPAN, as is known to occur in electrically conducting polymers. ${ }^{[15]}$ A reversible capacity of about $1500 \mathrm{~mA} \mathrm{~h}^{-1} \mathrm{~g}^{-1}$ (sulfur) was obtained in the second cycle, thus indicating a sulfur utilization approaching $90 \%$. Figure $2 b$ shows that the addition of TTFP remarkably affected the cycle stability of the pPAN@S composite. The
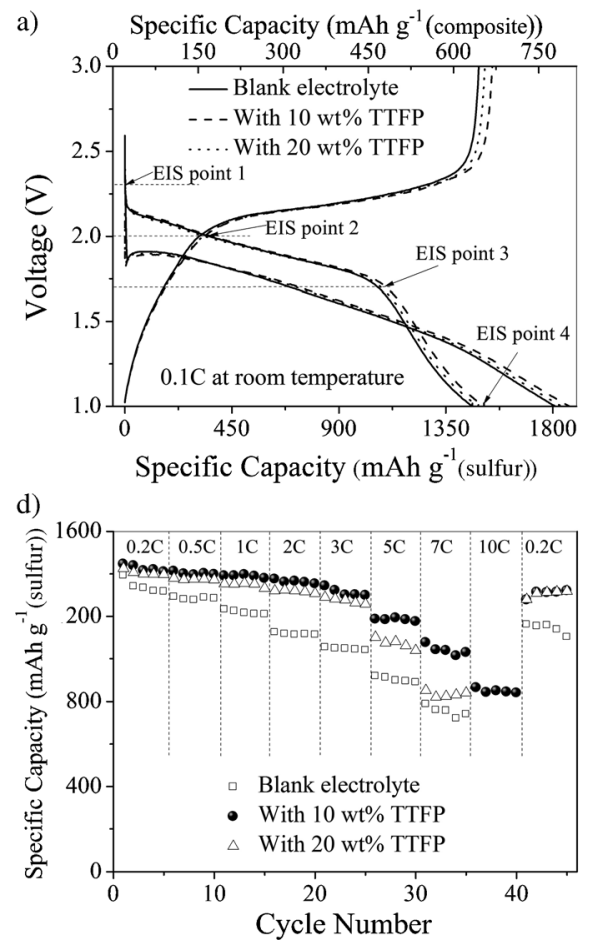

b)
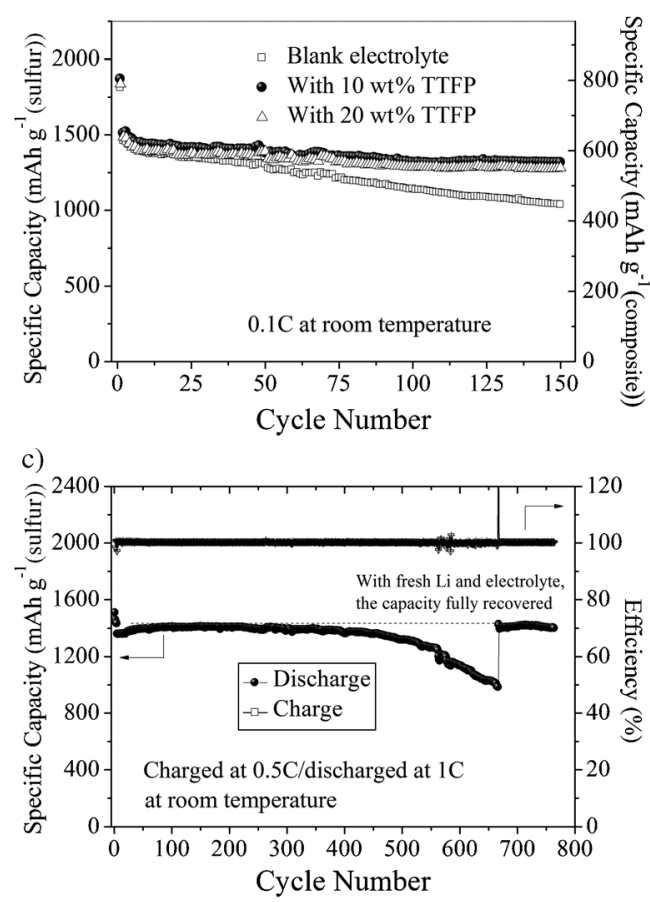

Figure 2. Electrochemical performance of pPAN@S composite cathodes in different electrolytes. a) Charge/discharge profiles; b) cycling performance; c) long-term cycling behavior in flame-inhibiting 
capacity gradually decayed in the blank electrolyte $(1 \mathrm{M} \mathrm{LiPF} /$ $\mathrm{EC}+\mathrm{DMC}$ ), with only $1037 \mathrm{~mA} \mathrm{~h}^{-1} \mathrm{~g}^{-1}$ (sulfur) left after 150 cycles. In contrast, the composite cathode in the electrolyte containing TTFP demonstrated a stable cycling performance apart from the initial 3 cycles. During long-term cycling, no remarkable capacity decrease was observed before 400 cycles, but the capacity decreased rapidly after 500 cycles.

After 666 cycles, the pPAN@S cathode was removed and was reassembled in a new cell with a fresh $\mathrm{Li}$ anode and new electrolyte. The capacity of the pPAN@S cathode fully recovered to its initial value of $1400 \mathrm{~mA} \mathrm{~h}^{-1} \mathrm{~g}^{-1}$ (sulfur) (Figure $2 \mathrm{c}$ ), with no degradation of the performance except for a slight increase in the charge voltage (see Figure S1 in the Supporting Information). Therefore, the rapid decline in the capacity after 500 cycles was ascribed to the lithium-metal anode, which had two critical problems: lithium dendrite formation and the occurrence of side reactions during cycling, which led to high internal resistance as the lithium was pulverized and the electrolyte was consumed.$^{[16]}$ Notably, the pPAN@S composite exhibited an extremely stable cycling behavior in the nonflammable electrolyte, with no degradation in the capacity after 750 cycles or beyond.

The high power rate performance also benefited from the addition of TTFP. In the blank electrolyte, the pPAN@S composite delivered only about $740 \mathrm{~mA} \mathrm{~h}^{-1} \mathrm{~g}^{-1}$ (sulfur) at $7 \mathrm{C}$ (where $1 \mathrm{C}$ is equal to a current density of $1672 \mathrm{mAg}^{-1}$ (sulfur)). The addition of $10 \mathrm{wt} \%$ TTFP
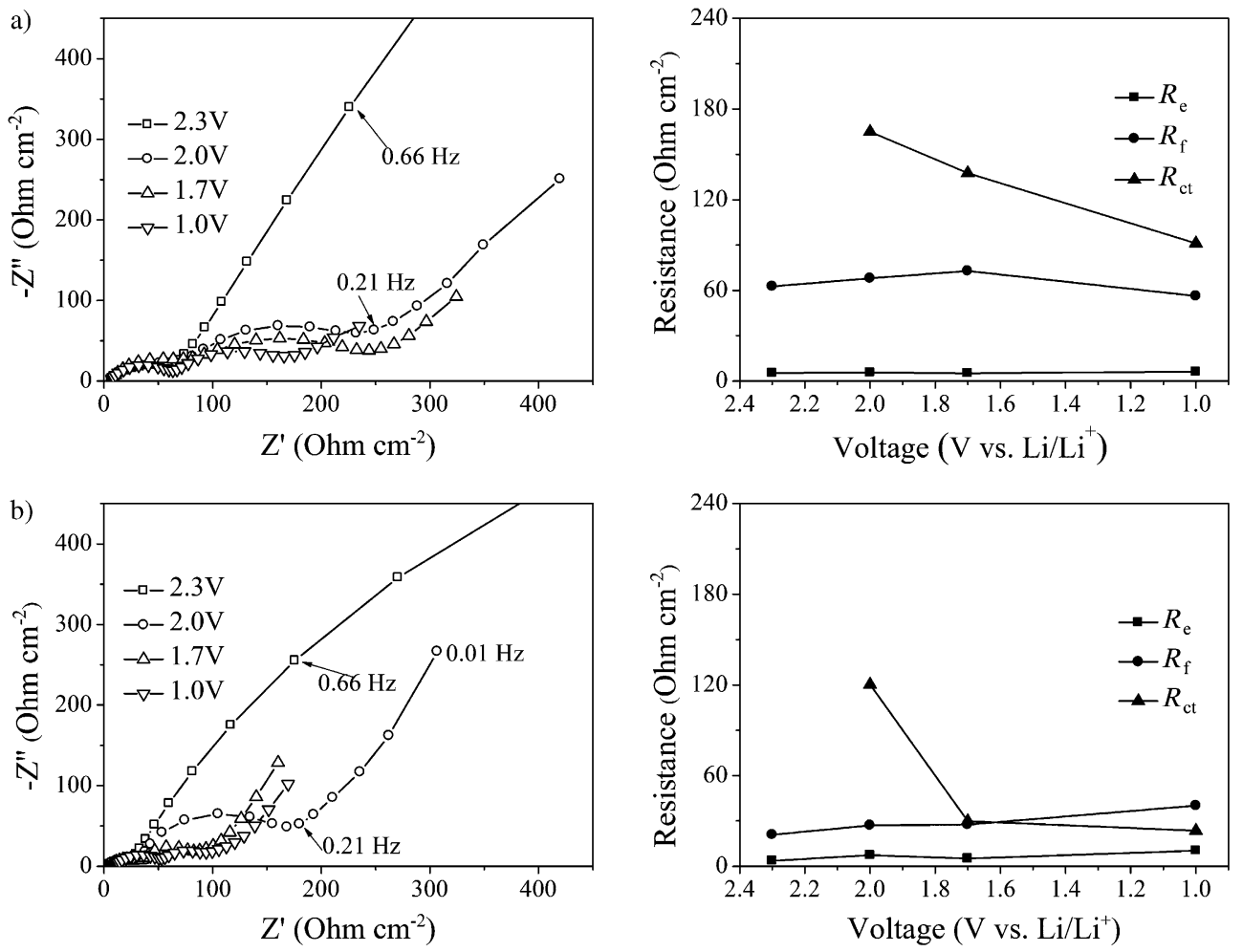

Figure 3. EIS results at different voltages and the corresponding electrochemical parameters. a) Blank electrolyte; b) blank electrolyte +10 wt \% TTFP. cathodes exhibited high coulombic efficiencies approaching $100 \%$, except in the first discharge process, in all the electrolytes.

To explain the impact of TTFP on the performance of the pPAN@S composite cathode, the electrochemical impedance spectra (EIS) of the cathode were measured at discharge the cathode remained in a fully charged state (Figure 2 a). Only one semicircle in the high- and medium-frequency regions and a sloping line in the low-frequency region were observed Figure S3 in the Supporting Information). The other impedance spectra are composed of two overlapped semicircles and a sloping line (Figure 3, left).

\section{to the electrolyte not only}

significantly enhanced the cycle capacity at different current rates but also suppressed the voltage polarization (Figure $2 \mathrm{~d}$ and see Figure S2 in the Supporting Information). With TTFP additives of $10 \mathrm{wt} \%$ and $20 \mathrm{wt} \%$, the pPAN@S composite delivered similar capacities at discharge rates up to $3 \mathrm{C}$; afterwards, a difference in capacity became distinct. Even when discharged at $10 \mathrm{C}$ in $10 \mathrm{wt} \%$ TTFP electrolyte, the capacity was maintained at about $850 \mathrm{~mA} \mathrm{~h}^{-1} \mathrm{~g}^{-1}$ (sulfur). The clear decreases in capacity observed at high discharge rates for $20 \mathrm{wt} \%$ TTFP are attributed to the high viscosity and low dielectric constant of TTFP. After the rate was decreased to $0.2 \mathrm{C}, 87 \%$ of the initial capacity was recovered in the case of the blank electrolyte, whereas $94 \%$ was recovered in the case of the nonflammable electrolytes. Moreover, the composite
The EIS were fitted by the equivalent circuits (see Figure S4 in the Supporting Information), and the results are given in Figure 3 (right) (the detailed electrochemical parameters are listed in Table S1 in the Supporting Information). The addition of TTFP clearly decreased both the lithium-ion transfer resistance $\left(R_{\mathrm{f}}\right)$ in the interfacial film and the electrochemical reaction resistance $\left(R_{\mathrm{ct}}\right)$. We speculate that TTFP might participate in interfacial reactions on the cathode to form a film that enhances lithium-ion transfer and accelerates the electrochemical reactions.

As the rate performance is closely associated with the diffusion of $\mathrm{Li}^{+}$ions in the electrode materials, it was necessary to investigate the impact of TTFP on $\mathrm{Li}^{+}$diffusion. The lithium-ion diffusion coefficient was evaluated by cyclic 
voltammetry $(\mathrm{CV})$ and was calculated according to the Randles-Sevick Equation [Eq. (1)]: ${ }^{[17]}$

$I_{\mathrm{p}}=2.69 \times 10^{5} n^{1.5 \mathrm{AD}} \mathrm{D}_{\mathrm{Li}}^{0.5} v^{0.5} \mathrm{C}_{\mathrm{Li}}=B \times{ }^{v^{0.5}}$

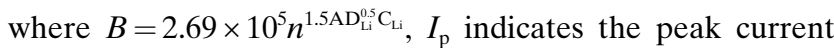
(A), $\mathrm{C}_{\mathrm{Li}}$ is the lithium-ion concentration in the electrolyte, $\mathrm{A}$ is the electrode area (here, $1.13 \mathrm{~cm}^{2}$ ), $v$ is the scanning rate $\left(\mathrm{Vs}^{-1}\right), n$ is the number of electrons in the reaction (here, 2; $\left.2 \mathrm{Li}+\mathrm{S} \rightarrow \mathrm{Li}_{2} \mathrm{~S}\right)$, and $\mathrm{D}_{\mathrm{Li}}{ }^{+}$is the lithium-ion diffusion coefficient $\left(\mathrm{cm}^{2} \mathrm{~s}^{-1}\right)$.

According to Equation (1), the plot of the oxidation (or reduction) peak current $I_{\mathrm{p}}$ versus the square root of the scan rate $\left(v^{0.5}\right)$ should result in a straight line. Figure $4 \mathrm{c}$ demonstrates that $I_{\mathrm{p}}$ was indeed proportional to ${ }^{v^{0.5}}$, thus confirming

$10^{-9}$ Torr overnight, which ensured that no TTFP liquid remained in the cathodes (the boiling point of TTFP is approximately $131^{\circ} \mathrm{C}$ ). The XPS spectrum of the cathode cycled in the nonflammable electrolyte showed a new $\mathrm{F} 1 \mathrm{~s}$ peak at $688.7 \mathrm{eV}$; this peak is associated with $\mathrm{CF}_{3}$ derived from TTFP (Figure 4d), which supports our speculation that TTFP competes with the blank electrolyte to participate in interfacial reactions on the cathode. The changes in the interfacial film conferred several advantages, including lower lithium diffusion resistance, accelerated electrochemical reactions, and partial accommodation of the volume change during cycling, ${ }^{[18]}$ thus resulting in a dramatically improved performance of the pPAN@S composite cathode. Moreover, the good wettability of TTFP provided intimate contact between the cathode and the electrolyte, which also favored reversible electrochemical reactions and long-term cycling stability.

When the pPAN@S composite was cycled in carbonate electrolytes, the surface sulfur first reacted with lithium to form polysulfides, $\mathrm{LiS}_{x}$ $(4 \leq x \leq 8)$. These polysulfides exhibited high solubility and strong electron-donating abilities. They reacted with carbonate solvents to form an interface that hindered the electrolyte from permeating into the interior of the pPAN@S composite. ${ }^{[5 b]}$ The disappearance of the $\mathrm{N} 1 \mathrm{~s}$ peak from the spectrum of the cathode cycled in the blank electrolyte clearly indicates the formation of an interface with a thickness beyond the detecting depth of XPS (typically several nanometers). In contrast, the weak $\mathrm{N}$ spectrum of the cathode cycled in the nonflammable electrolyte most

a diffusion-controlled mechanism. From the slope (B) of the linear fit, the lithium diffusion coefficients were calculated as $\mathrm{C}_{1}=6.79 \times 10^{-10}$ and $\mathrm{A}_{1}=3.243 \times 10^{-10}$ as well as $\mathrm{C}_{2}=9.455 \times$ $10^{-9}$ and $\mathrm{A}_{2}=5.673 \times 10^{-9}$, which correspond to the cathodic and anodic reactions in the blank and nonflammable electrolytes, respectively (see Table S2 in the Supporting Information). Although only the average values of $\mathrm{D}_{\mathrm{Li}}{ }^{+}$could be obtained using $\mathrm{CV}$, the average values are sufficient in this case to evaluate the effect of TTFP on $\mathrm{D}_{\mathrm{Li}}{ }^{+}$. It is clear that the addition of TTFP dramatically enhanced the lithium diffusion coefficient more than tenfold.

X-ray photoelectron spectroscopy (XPS) was used to reveal the changes in the interface after the addition of TTFP. Before the measurements, the cycled cathodes were washed in DMC three times and dried under a vacuum of about $5 \times$ likely indicates a thinner interface (see Figure S5a in the Supporting Information). For further electrochemical reactions, the pPAN main chain, with an electrical conductivity greater than $10^{-4} \mathrm{~S} \mathrm{~cm}^{-1}$, provides channels for the transfer of $\mathrm{Li}$ ions and electrons; monodispersed sulfur molecules, such as $\mathrm{S}_{4}, \mathrm{~S}_{2}$, or even $\mathrm{S}$ atoms, remain in the pPAN matrices and act as electrochemically active points. ${ }^{[19]}$ This novel structure theoretically ensures a high electrochemical activity, good reversibility, and long-term cycle stability of the sulfur composite cathode. No discharge products dissolved into the electrolyte (see Figure S5b in the Supporting Information).

Practically, because of the strong tendency of sulfur to sublime, residual elemental sulfur will inevitably adsorb onto the surface of the pPAN@S composite during the cooling 
process. The surface structure, or interfacial film formed between the pPAN@S and the carbonate electrolyte, determines the electrochemical performance of the pPAN@S composite cathode to a large extent. Zhang et al. reported that TTFP was initially reduced below $1.8 \mathrm{~V}$ to form a stable solid electrolyte interface (SEI) which protected the propylene carbonate (PC) from being decomposed on the graphite anode. ${ }^{[7 b]}$ For our Li-S battery, the voltage of the pPAN@S cathode during its first discharge process is mainly below $1.8 \mathrm{~V}$ (Figure 2a). Therefore, we speculate that TTFP is reduced during the first discharge to form a stable interfacial film on the pPAN@S cathode which prevents the reaction between EC and polysulfides, or, at least, affects their interaction. The detailed interfacial reaction mechanism requires further investigation through in situ or in operando techniques. ${ }^{[20]}$

In summary, we report a safe Li-S battery that exhibits an extremely stable cycling performance and an outstanding high-rate capability. The flammability issue of the Li-S battery was eliminated by using flame-inhibiting carbonate electrolytes derived from the addition of TTFP and a pPAN@S composite cathode with unique nonflammable properties. TTFP not only served as a flame retardant but also modified the interfacial film on the pPAN@S cathode that enhanced the Li-ion diffusion coefficient more than 10-fold and accelerated the electrochemical reactions. The pPAN@S composite cathode maintained extremely stable electrochemical activity over 750 cycles and exhibited a discharge capacity greater than $800 \mathrm{~mA} \mathrm{~h}^{-1} \mathrm{~g}^{-1}$ (sulfur) at a high rate of $10 \mathrm{C}$ in the flame-inhibiting electrolyte. The use of both a nonflammable electrolyte and cathode will allow the safety issues of Li-S batteries to be solved after the dendrite formation and low coulombic efficiency (CE) of the lithium-metal anode are addressed. ${ }^{[16 b, 21]}$ We hope that the exceptional results presented herein will speed the development and applications of safe and highly efficient beyond-lithium-ion batteries in the fields of renewable energy as well as in the wearable products. $^{[22]}$

\section{Experimental Section}

Cathode preparations and electrochemical measurements: The pPAN@S composite and cathode were prepared according to the method described previously. ${ }^{[19 a]}$ PAN was mixed homogeneously with elemental sulfur and heated at $300^{\circ} \mathrm{C}$ in a $\mathrm{N}_{2}$ atmosphere, thereby resulting in a pPAN@S composite as a black powder with a sulfur content of $43 \mathrm{wt} \%$, as determined by elemental analysis using a PE 2400 II CHNS/O elemental analysis system (PerkinElmer). The cathode was fabricated by mixing $80 \mathrm{wt} \%$ pPAN@S composite, $10 \mathrm{wt} \%$ Super P, and $10 \mathrm{wt} \%$ carbonyl- $\beta$-cyclodextrin as a binder, then coating the mixture onto a piece of $\mathrm{Al}$ foil (with a carbon coating) and cutting the foil into small disks with a diameter of $12 \mathrm{~mm}$; the weight loads of the foil disks were ca. $2.0 \mathrm{mg} \mathrm{cm}^{-2}$. The anode consisted of Li foil with a diameter of $16 \mathrm{~mm}$ and a thickness of $0.2 \mathrm{~mm}$. A $1 \mathrm{M} \mathrm{LiPF} / \mathrm{EC}+\mathrm{DMC}(1: 1, \mathrm{v} / \mathrm{v})$ solution was used as a blank electrolyte, in which tris(2,2,2-trifluoroethyl) phosphite (TTFP; Aldrich) was added directly at ratios of 5, 10, 15, and $20 \mathrm{wt} \%$. The charge/discharge cycles were performed in a cut-off voltage limit of $1.0-3.0 \mathrm{~V}$ on a LAND cycler (Wuhan, China).

Electrochemical impedance spectra (EIS) and cyclic voltammetry (CV): To measure the electrochemical kinetics of the sulfur electrode, three-electrode electrochemical cells were assembled in a glove box (MBraun, Germany) using lithium foil as the counter electrode, a lithium ring as the reference electrode, the pPAN@S cathode as the working electrode, and the PE film as a separator. After 3 cycles at $0.1 \mathrm{C}, \mathrm{CV}$ and EIS were performed using a Solartron FRA 1250 frequency response analyzer in combination with a Solartron SI 1287 electrochemical interface. The frequency ranged from $100 \mathrm{kHz}$ to $0.01 \mathrm{~Hz}$, and the amplitude was set at $5 \mathrm{mV}$. The scan rate for $\mathrm{CV}$ was increased gradually from 0.1 to $0.4 \mathrm{mV} \mathrm{s}^{-1}$ in a voltage range of 1.0 to $3.0 \mathrm{~V}$ versus $\mathrm{Li} / \mathrm{Li}^{+}$. All the experiments were conducted at room temperature.

See the Supporting Information for the measurements of the selfextinguishing time (SET), conductivity, and X-ray photoelectron spectroscopy (XPS).

Received: May 10, 2014

Published online: July 24, 2014

Keywords: batteries - electrochemistry

flame-inhibiting electrolyte · lithium · sulfur

[1] a) X. L. Ji, K. T. Lee, L. F. Nazar, Nat. Mater. 2009, 8, 500-506; b) Z. W. She, W. Y. Li, J. J. Cha, G. Y. Zheng, Y. Yang, M. T. McDowell, P. C. Hsu, Y. Cui, Nat. Commun. 2013, 4, 1331; c) N. Jayaprakash, J. Shen, S. S. Moganty, A. Corona, L. A. Archer, Angew. Chem. 2011, 123, 6026-6030; Angew. Chem. Int. Ed. 2011, 50, 5904-5908; d) Y. Z. Fu, Y. S. Su, A. Manthiram, Angew. Chem. 2013, 125, 7068-7073; Angew. Chem. Int. Ed. 2013, 52, 6930-6935.

[2] R. Van Noorden, Nature 2014, 507, 26-28.

[3] X. L. Ji, L. F. Nazar, J. Mater. Chem. 2010, 20, 9821-9826.

[4] Y. X. Yin, S. Xin, Y. G. Guo, L. J. Wan, Angew. Chem. 2013, 125, 13426-13441; Angew. Chem. Int. Ed. 2013, 52, 13186-13200.

[5] a) J. L. Wang, J. Yang, J. Y. Xie, N. X. Xu, Adv. Mater. 2002, 14, $963-965$; b) J. Gao, M. A. Lowe, Y. Kiya, H. D. Abruna, J. Phys. Chem. C 2011, 115, 25132-25137.

[6] O. S. Bushuyev, P. Brown, A. Maiti, R. H. Gee, G. R. Peterson, B. L. Weeks, L. J. H. Weeks, J. Am. Chem. Soc. 2012, 134, $1422-$ 1425.

[7] a) K. Xu, S. S. Zhang, J. L. Allen, T. R. Jow, J. Electrochem. Soc. 2002, 149, A1079-A1082; b) S. S. Zhang, K. Xu, T. R. Jow, Electrochem. Solid-State Lett. 2002, 5, A206-A208.

[8] a) J. Hassoun, B. Scrosati, Adv. Mater. 2010, 22, 5198-5201; b) Z. Lin, Z. C. Liu, W. J. Fu, N. J. Dudney, C. D. Liang, Angew. Chem. 2013, 125, 7608-7611; Angew. Chem. Int. Ed. 2013, 52, $7460-$ 7463; c) J. Dong, Z. Zhang, Y. Kusachi, K. Amine, J. Power Sources 2011, 196, 2255-2259; d) J. W. Park, K. Ueno, N. Tachikawa, K. Dokko, M. Watanabe, J. Phys. Chem. C 2013, 117, $20531-20541$.

[9] B. B. Wu, F. Pei, Y. Wu, R. J. Mao, X. P. Ai, H. X. Yang, Y. L. Cao, J. Power Sources 2013, 227, 106-110.

[10] E. P. Roth, C. J. Orendorff, Electrochem. Soc. Interface 2012, 21, $45-49$.

[11] J. Zhang, G. W. H. Silcock, T. J. Shields, J. Fire Sci. 1995, 13, 141 161.

[12] L. C. Yin, J. L. Wang, F. J. Lin, J. Yang, Y. N. Nuli, Energy Environ. Sci. 2012, 5, 6966-6972.

[13] Z. Bashir, Carbon 1991, 29, 1081-1091.

[14] a) L. Shi, H. W. Liu, P. Xu, D. F. Zhao, Adv. Mater. Res. 2011, 175-176, 465-468; b) C. Liu, S. L. Zhao, J. Tianjin Polytech. Univ. 2011, 30, 29-31.

[15] a) J. L. Bredas, R. Silbey, D. S. Boudreaux, R. R. Chance, J. Am. Chem. Soc. 1983, 105, 6555-6559; b) L. W. Shacklette, J. E. Toth, N. S. Murthy, R. H. Baughman, J. Electrochem. Soc. 1985, $132,1529-1535$. 


\section{Angewandte}

Communications

[16] a) D. Aurbach, E. Zinigrad, H. Teller, P. Dan, J. Electrochem. Soc. 2000, 147, 1274-1279; b) W. Xu, J. L. Wang, F. Ding, X. L. Chen, E. Nasybulin, Y. H. Zhang, J. G. Zhang, Energy Environ. Sci. 2014, 7, 513-537.

[17] a) H. J. Jung, J. Hassoun, J. B. Park, Y. K. Sun, B. Scrosati, Nat Chem. 2012, 4, 579-585; b) S. R. Das, S. B. Majumder, R. S. Katiyar, J. Power Sources 2005, 139, 261-268.

[18] X. M. He, J. G. Ren, L. Wang, W. H. Pu, C. Y. Jiang, C. R. Wan, J. Power Sources 2009, 190, 154-156.

[19] a) J. L. Wang, Z. D. Yao, C. W. Monroe, J. Yang, Y. N. Nuli, $A d v$. Funct. Mater. 2013, 23, 1194-1201; b) J. Fanous, M. Wegner, J.
Grimminger, A. Andresen, M. R. Buchmeiser, Chem. Mater. 2011, 23, 5024-5028.

[20] X. S. Liu, D. D. Wang, G. Liu, V. Srinivasan, Z. Liu, Z. Jussain, W. L. Yang, Nat. Commun. 2013, 4, 2568.

[21] M. S. Park, S. B. Ma， D. J. Lee, D. M. Im, S. G. Doo, O. Yamamoto, Sci. Rep. 2014, 4, 3815.

[22] a) M. Koo, K. Park, S. H. Lee, M. Suh, D. Y. Jeon, J. W. Choi, K, Kang, K. J. Lee, Nano Lett. 2012, 12, 4810-4816; b) Z. Song, T. Ma, R. Tang, Q. Cheng, X. Wang, D. Krishnaraju, R. Panat, C. K. Chan, H. Yu, H. Q. Jiang, Nat. Commun. 2014, 5, 3140. 